University of Wollongong

Research Online

Faculty of Engineering and Information

Faculty of Engineering and Information

Sciences - Papers: Part A

Sciences

2014

\title{
A comparative analysis of multichannel data acquisition systems for quality assurance in external beam radiation therapy
}

Iolanda Fuduli

University of Wollongong, if473@uowmail.edu.au

Claudiu Porumb

University of Wollongong, csp528@uowmail.edu.au

Anthony Espinoza

University of Wollongong, aae718@uowmail.edu.au

Abdullah Aldosari

University of Wollongong, ahaa215@uowmail.edu.au

Martin Carolan

University of Wollongong, mcarolan@uow.edu.au

See next page for additional authors

Follow this and additional works at: https://ro.uow.edu.au/eispapers

Part of the Engineering Commons, and the Science and Technology Studies Commons

Research Online is the open access institutional repository for the University of Wollongong. For further information contact the UOW Library: research-pubs@uow.edu.au 


\title{
A comparative analysis of multichannel data acquisition systems for quality assurance in external beam radiation therapy
}

\author{
Abstract \\ The paper presents a comparative study performed by the Centre of Medical Radiation Physics (CMRP) \\ on three multichannel Data Acquisition Systems (DAQ) based on different analogue front-ends to suit a \\ wide range of radiotherapy applications. The three front-ends are: a charge-to-frequency converter \\ developed by INFN Torino, an electrometer and a charge-to-digital converter (both commercial devices \\ from Texas Instruments). For the first two (named DAQ A and B), the CMRP has designed the read-out \\ systems whilst the third one (DAQ C) comes with its own evaluation board. For the purpose of the \\ characterization DAQ A and DAQ B have been equipped with 128 channels while DAQ $C$ has 256 channels. \\ In terms of performances, the DAQs show good linearity over all the dynamic range. Each one has a \\ different range of sensitivity ranging from less than $1 \mathrm{pC}$ up to $13 \mathrm{nC}$, which makes the three front-ends \\ complementary and suitable for use with different radiation detectors for different radiotherapy \\ applications, or in a mixed solution which can house different front-ends.
}

\section{Keywords}

beam, comparative, radiation, analysis, therapy, multichannel, data, acquisition, systems, quality, assurance, external

\section{Disciplines \\ Engineering | Science and Technology Studies}

\section{Publication Details}

Fuduli, I., Porumb, C., Espinoza, A., Aldosari, A., Carolan, M., Lerch, M. LF., Metcalfe, P. E., Rosenfeld, A. \& Petasecca, M. (2014). A comparative analysis of multichannel data acquisition systems for quality assurance in external beam radiation therapy. Journal of Instrumentation, 9 (6), 1-12.

\section{Authors}

Iolanda Fuduli, Claudiu Porumb, Anthony Espinoza, Abdullah Aldosari, Martin Carolan, Michael LF Lerch, Peter E. Metcalfe, Anatoly Rosenfeld, and Marco Petasecca 


\title{
A comparative analysis of multichannel Data Acquisition Systems for Quality Assurance in external beam radiation therapy
}

\author{
I. Fuduli ${ }^{a}$, C. Porumb ${ }^{a}$, A.A. Espinoza ${ }^{a}$, A.H. Aldosari ${ }^{a}$, M.Carolan ${ }^{a, b}$, M.L.F.

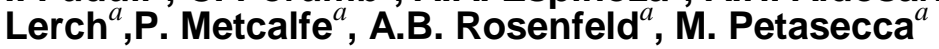 \\ ${ }^{a}$ Centre for Medical Radiation Physics, \\ University of Wollongong, NSW 2522, Australia \\ ${ }^{b}$ Illawarra Cancer Care Centre, \\ Wollongong Hospital, NSW 2500, Australia \\ E-mail: if473@uowmail.edu.au
}

ABSTRACT: The paper presents a comparative study performed by the Centre of Medical Radiation Physics (CMRP) on three multichannel Data Acquisition Systems (DAQ) based on different analogue front-ends to suit a wide range of radiotherapy applications. The three frontends are: a charge-to-frequency converter developed by INFN Torino, an electrometer and a charge-to-digital converter (both commercial devices from Texas Instruments). For the first two (named DAQ A and B), the CMRP has designed the read-out systems whilst the third one (DAQ C) comes with its own evaluation board. For the purpose of the characterization DAQ A and DAQ B have been equipped with 128 channels while DAQ C has 256 channels. In terms of performances, the DAQs show good linearity over all the dynamic range. Each one has a different range of sensitivity ranging from less than $1 \mathrm{pC}$ up to $13 \mathrm{nC}$, which makes the three front-ends complementary and suitable for use with different radiation detectors for different radiotherapy applications, or in a mixed solution which can house different front-ends.

KEYWORDS: Front-end electronics for detector readout; Data acquisition concepts; Dosimetry concepts and apparatus. 


\section{Contents}

1. Introduction $\quad 1$

2. Materials and Methods 3

2.1 Graphical User Interface $\quad 3$

2.2 Front end TERA06 4

2.3 Front end AFE0064

2.4 Front end DDC264

3. Experimental results and discussion $\quad 6$

4. Conclusion $\quad 9$

\section{Introduction}

New radiotherapy cancer treatment techniques such as Intensity Modulated Radiation Therapy (IMRT), Volumetric Modulated Arc Therapy (VMAT), Tomotherapy and Stereotactic Radiosurgery (SRS) use a radiation beam to target the treatment of the affected volume within the body of the patient. The increasing complexity of treatment delivery has enabled the escalation of the target dose, a reduction in the margins of the treatment field sizes and increase in the dose per fraction, while maintaining side effects to the surrounding organs-at-risks at an acceptable level [1]. However, such higher conformity and hypofractionation have also created the conditions for more severe undesired effects in the case of a malfunctioning medical accelerator or human error during the planning phase or the patient treatment. Therefore, the role of quality assurance (QA) and treatment is becoming extremely important. 2D dose distributions are generally evaluated by several means including passive (e.g. radiochromic films, TLDs) and active systems (e.g. electronic portal imaging devices or electronic equipment based on small volume ionising chambers or silicon diodes). The electronic devices for 2D or 3D QA in external beam radiotherapy are based on multichannel pixellated detector connected to a front-end which feeds the analog to digital data converters. The requirements for the detectors are high spatial resolution and high sensitivity. The readout system needs also to have a wide dynamic range and good linearity.

Currently there are multiple commercially available instruments which have been designed for 2D and 3D dose mapping for External Beam Radiation Therapy (EBRT) such as Octavius 4D from PTW, ArcCHECK from Sun Nuclear and Delta ${ }^{4}$ from ScandiDos. Octavius 4D is a rotating phantom which can house a detector plate based on ionising chambers [2]. It has the capability to keep the detector always perpendicular to the beam using an inclinometer placed onto the LINAC gantry as an independent position monitoring of the head. The system has no dead time and it communicates with the PC via RS232 or Ethernet protocol. The minimum acquisition time frame of the sensor array is limited to $200 \mathrm{~ms}$. ArcCHECK instead has a builtin helical grid of SunPoint diode detectors; the angle of the gantry is measured by an 


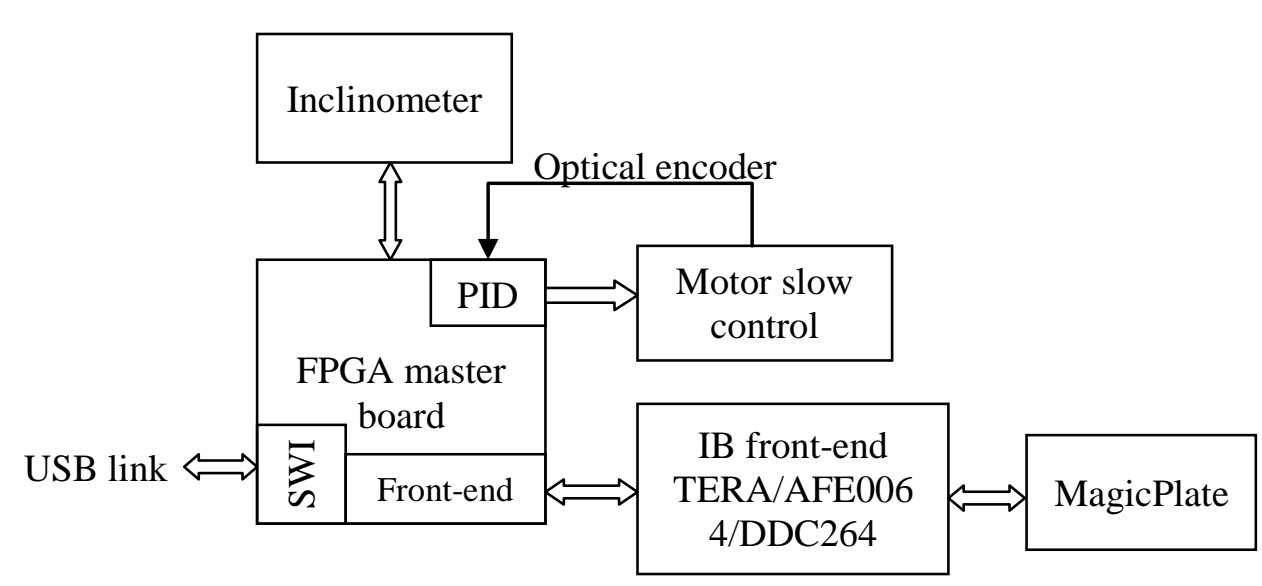

Figure 1: DAQ schematic block diagram; The IB module identifies the three options of the front-end electronics investigated in this work

inclinometer and embedded in the calculations performed by the software [3]. Delta ${ }^{4}$ represents another example of QA equipment based on silicon diodes; sensors are arranged in two 2D arrays of 1069 elements distributed across $20 \times 20 \mathrm{~cm}^{2}$ of sensitive area. The orientation of the phantom can be adjusted by the user but the 3D dose measure is calculated by the software. The acquisition frame is synchronised with the LINAC and integration of the current is performed only during the beam pulse (i.e. $360 \mathrm{~Hz}$ for a Varian $2100 \mathrm{EX}$ linear accelerator). The system communicates to the PC via a proprietary protocol through a Cat-5 cable. All the commercial instruments are competitive for an end-user clinical environment, but most of their Data Acquisition Systems (DAQ) do not allow access to the information at a very low level, such single channel response of the detector as a function of time, or the use of different detectors, important features for research purposes. The Centre of Medical Radiation Physics (CMRP) has designed a custom DAQ. Several multichannel architectures have been investigated, each one based on different analogue front-end to suit a wide range of applications in radiotherapy, from pre-treatment QA in a phantom to intra-operative beam monitoring device such as MagicPlate [4]. The aim of this study is the characterisation and comparison of the performance of three solutions based on research grade and commercial electrometer chips for use in 2D/3D and 4D dose mapping in EBRT. The DAQ ultimate goal is the readout of two 2D MagicPlate detectors, each of them constituted by an array of $11 \times 11$ CMRP p-type diodes with a sensitive volume of $0.6 \times 0.6 \times 0.05 \mathrm{~mm}^{3}$ and detector pitch of $10 \mathrm{~mm}$. The detector has been fully characterised by Wong et al. [4] with reproducibility within $1.1 \%$, percentage depth dose compared to ionisation chamber within $0.7 \%$ and good dose linearity. The detector is inserted in a rotating phantom that follows the movements of the head of the LINAC to be always perpendicular to the beam and avoid complex correction algorithms of the diode's angular dependence. The second detector is instead mounted on the LINAC accessory tray for online beam monitoring during the treatment.

The DAQ has 256 channels and is controlled by a custom designed FPGA Master Board (FPGA MB) communicating with the PC through a USB2.0 link.

Figure 1 shows the schematic block diagram of the DAQ architecture. The FPGA MB houses a Xilinx XC3S400 and the Cypress USB interface. The MB drives the inclinometer, located on the accessory tray with the MagicPlate beam monitoring detector, and gives the position of the gantry head to align the phantom with the beam. A proportional-integralderivative (PID) controller module has been designed and implemented in the FPGA to set and 
Table 1: Data Acquisition Systems Specifications

\begin{tabular}{|l|l|c|c|c|c|}
\hline Parameter & \multicolumn{1}{|c|}{ Description } & DAQ A & DAQ B & $\begin{array}{c}\text { DAQ C } \\
\text { (evaluation } \\
\text { board })\end{array}$ & Unit \\
\hline $\begin{array}{l}\text { Num. of } \\
\text { channels }\end{array}$ & Number of channel acquired & $128 / 256$ & $128 / 256$ & 256 & \\
\hline Resolution & Analog to Digital conversion & 16 & 16 & $16 / 20$ & bit \\
\hline Dead Time & Time needed for data transfer & $14 / 28$ & 67 & $/$ & $\mu \mathrm{s}$ \\
\hline $\begin{array}{l}\text { Minimum } \\
\text { IT }\end{array}$ & Minimum Integration Time & 1 & 0.014 & 0.166 & $\mathrm{~ms}$ \\
\hline MSR & Maximum frame sampling rate & 1 & 10 & 6 & $\mathrm{kHz}$ \\
\hline MCR & Maximum conversion rate & 10 & 1 & 0.003 & $\mathrm{MHz}$ \\
\hline BW & Bandwidth of the DAQ & 23 & 160 & 3 & $\mathrm{kHz}$ \\
\hline
\end{tabular}

control the position of the phantom based on the information that the inclinometer and the optical encoder provide. A Graphical User Interface (GUI) has been custom designed by CMRP and provides the operator with all the controls to acquire the data from the detector, to manage the phantom rotation and to monitor the LINAC gantry position, in real time. Three multichannel devices have been characterised and tested for the use with MagicPlate: the TERA06 charge-to-frequency converter (DAQ A), an ASIC developed to readout an array of ionisation chambers used in radiation therapy [5]; the commercial electrometer named AFE0064 (DAQ B) from Texas Instrument (TI) and the multichannel charge-to-digital converter from TI called DDC264 (DAQ C). For the purpose of the comparison with the TERA and the AFE, the DDC264 has been tested in terms of full scale range, noise and resolution using its own evaluation board DDC264EVM (TI) [6], which is equipped with a Xilinx Spartan3 FPGA and a USB FDTI interface.

\section{Materials and Methods}

In order to compare the performances of the three DAQ architectures (Tab.1) an array of 128 resistors polarised by a waveform generator has been used to simulate the current generated by a pulsed radiation source such as a medical LINAC [7]. For this set of measurements, the following parameters have been defined: integration time (IT) as the interval of integration of the input current; acquisition frame (frame) as each set of 128/256 channels read simultaneously; dead time (DT) as the time interval required for the acquisition of the data during which the analog front-end is disabled.

For all the three architectures, the DAQ has been placed into a metallic enclosure with an aperture for the input resistor array to simulate the normal conditions of operation where the detector connected to the electronics is exposed to the beam. The DAQ is then connected to a laptop by 25 meters long USB2.0 extension cable.

\subsection{Graphical User Interface}

The GUI, named RadX-DoseView, has been entirely designed by CMRP. It has been compiled under the $\mathrm{C}++$ developing suite Nokia QT rev4.0. The GUI manages the USB link using dynamic language libraries (DLL) specifically developed for the Cypress chip interface. It initializes the USB-link, sends the firmware to the FPGA and acknowledges if the device is connected and fully operating. The operator masters the acquisition settings and the relevant parameters such as integration and the acquisition time and all the complementary signals 
necessary for the operation of each specific analogue front end. Commands are sent through the USB using specific endpoints defined into the firmware and dedicated to the communication of the triggers, the data, the channel headers and parity bits. Once the acquisition runs, the FPGA stores the data into an internal First-In First-Out memory stage (FIFO) and triggers the GUI to start the download of the data in burst-mode. The size of the burst can be user-defined and optimised based on the CPU computation load of the host computer to minimise the USB latency which varies between 2 to $3 \mathrm{~ms}$. Data losses are avoided by the use of a dual, cascade FIFO buffer stage of 17 Kbyte of RAM.

As the detector is comprised of multiple diodes each with its own intrinsic response to radiation, an important feature of the GUI is to acknowledge and take into account these minor intrinsic differences and to equalize the response. This is done by irradiating the detector with a $20 \times 20 \mathrm{~cm}^{2}$ broad beam by a $6 \mathrm{MV}$ photon beam at a depth of $10 \mathrm{~cm}$ in solid water. In this condition, the flatness of the LINAC beam is guarantee within the $2 \%$ fluctuation [8] and equalisation is then performed by using the response of the central detector as reference.

Though the GUI, the user can load the firmware into the FPGA; select the integration time, the duration of the acquisition, and the number of channels to be read out, the frequency of the readings (frames) and synchronised or asynchronous acquisitions by an external or an internally generated trigger, respectively. The limits of each parameter depend on the front end in use and are automatically updated by the interface after the handshake with the instrument. Data are decoded and stored in a file, named by the user, and each channel can be displayed in real-time or by a post-process analysis tab. The visualization can be changed according to the detector used and can be a histogram with counts versus channel, frame-by-frame integral, or in a colour mapping mode for 2D visualization. The measurement can also be visualised in playback as a colour map calibrated in dose (Gy) or counts. RadX-DoseView provides also the visualisation of the position of the LINAC gantry and of the rotatable phantom in real time.

\subsection{Front end TERA06}

The DAQ A houses two TERA06 chips to readout 128 channels simultaneously [9]. The interface board (IB) consists of a buffer shifter to match the TTL logic levels required for the TERA chip and provides the power supply rails. Each channel of the TERA06 is a charge to frequency converter, followed by a 16-bit counter. The input current from the detector $\left(\mathrm{I}_{\text {in }}\right)$ is integrated using an operational amplifier configured as an integrator with a capacitance $\mathrm{C}_{\text {int }}$. When the output of the integrator exceeds a given threshold $\mathrm{V}_{\mathrm{th}}$, a fixed amount of charge $\mathrm{Q}_{\mathrm{c}}$ (named reset charge) is subtracted from the capacitor $\mathrm{C}_{\text {int }}$ and a pulse is sent to the counter. The frequency of the pulse follows the relation $f=I_{i n} / Q_{c}$. As important feature, the subtraction of the $\mathrm{Q}_{c}$ reduces the output of the amplifier and resets the comparator without affecting the acquisition [8, 10]. The values of $\mathrm{V}_{\text {th }}$ and $\mathrm{Q}_{\mathrm{c}}$ are settable by the user through external potentiometers. The output of the TERA06 is a 16 bit digital number. When the FPGA sends a latch signal to the TERA06, it simultaneously stores the values of all the counters in a shift register and makes the output of the shift register available to be readout. A digital reset can be used for zeroing the counters. The TERA06 can be read in two modalities: Single Sampling (SS) and Double Sampling (DS). In SS, counters are reset after each read-out cycle, thus introducing a dead time during the transmission of the channels of approximately $14 \mu$ s for 128 channels. In DS, counters continuously accumulate the number of events, the value read must be subtracted by the previous sample and overflow will occur without notice. The two modalities have been investigated to estimate their effect on the measure of the charge. 


\subsection{Front end AFE0064}

The DAQ B houses two AFE0064 chips; each of them has 64 parallel input channels and two differential outputs. Once the appropriate command signals are sent to the chip, all the channels integrate the input current simultaneously. The charge accumulated for each channel is then converted in a voltage level and stored in an analogue buffer until the command for output sampling is sent and the information becomes available to be converted by the ADC. The differential outputs can be read in simultaneous mode, which means that they show a new value each clock pulse or in sequential mode, with the analogue outputs available alternatively every clock cycle [11].

The sequential mode has been chosen to match the sampling rate of the ADC which is a fully differential four channels 16-bit ADC from TI and which works in parallel with two AFEs. The architecture allows to readout all the channels in 133 clock cycles. When the outputs are enabled, the integrators are reset, thus introducing a total DT of about $70 \mu$ s using a $2 \mathrm{MHz}$ clock. The FPGA drives both the AFEs and the ADC, synchronising the devices by a custom designed internal clock manager. The ADC provides two distinguished serial outputs [12] with an effective output rate of 1 MSPS.

Modern medical LINACs are based on a pulsed radiation beam with repetition rate ranging from $200 \mathrm{~Hz}$ up to $400 \mathrm{~Hz}$ [7]; the electron gun trigger pulse, during which the beam is fired, lasts for $3.7 \mu$ s with a pulse period width of few milliseconds. The AFE0064 presents the major advantage to be able to sample the detector only when the beam is on, by the synchronisation of the detector sampling and the analogue to digital conversion with the LINAC. This modality has the double advantage of minimising the effects of electronic noise and leakage current, and managing the dead time by reading out the integrators while the beam is off. The DAQ B has been synchronised by the means of the synch signal provided by the LINAC which is a TTL signal synchronous with the maximum repetition rate of the electron gun trigger corresponding to the highest dose rate of the accelerator; this approach guarantees the pulse falls within the integration time avoiding any loss of charge. To give more flexibility to the system and be able to use it with LINACs from different manufacturers (Elekta, Varian or Siemens), the frequency can be adjusted by the user and goes up to $5 \mathrm{kHz}$. Dynamic range is also adjustable by the GUI by changing the full range scale from $0.13 \mathrm{pC}$ up to $9.6 \mathrm{pC}$ in eight steps. DAQ B has been tested using an array of $10 \mathrm{M} \Omega(1 \%$ accuracy) resistors polarized with a fixed current of $18 \mathrm{nA}$ and readout at the largest scale range available.

\subsection{Front end DDC264}

The DAQ C is based on the DDC264 EVM evaluation board provided by TI and equipped with 256 channels. The evaluation board has been modified and integrated into the architecture of the DAQ to be readout by the FPGA and controlled by the GUI.

The operator interface allows the selection of the frequency of the acquisition, the full scale range and the IT, it shows the results in real-time and provides data analysis features. The evaluation board comes also with a set of 256 resistors (10 M $\Omega$ ) which can be polarized using an external voltage signal. The DDC264 provides two integrators for each channel; when the first is active the second is off and transfers the value of the potential generated by the charge accumulated during the IT across the integration capacitor to the digitalisation stage. Therefore, DAQ C does not have dead time during the acquisition, which is particularly important for application where the radiation source is not pulsed. The full scale range extends from $12.5 \mathrm{pC}$ up to $150 \mathrm{pC}$, making the DAQ C very promising for high dose rate applications. 

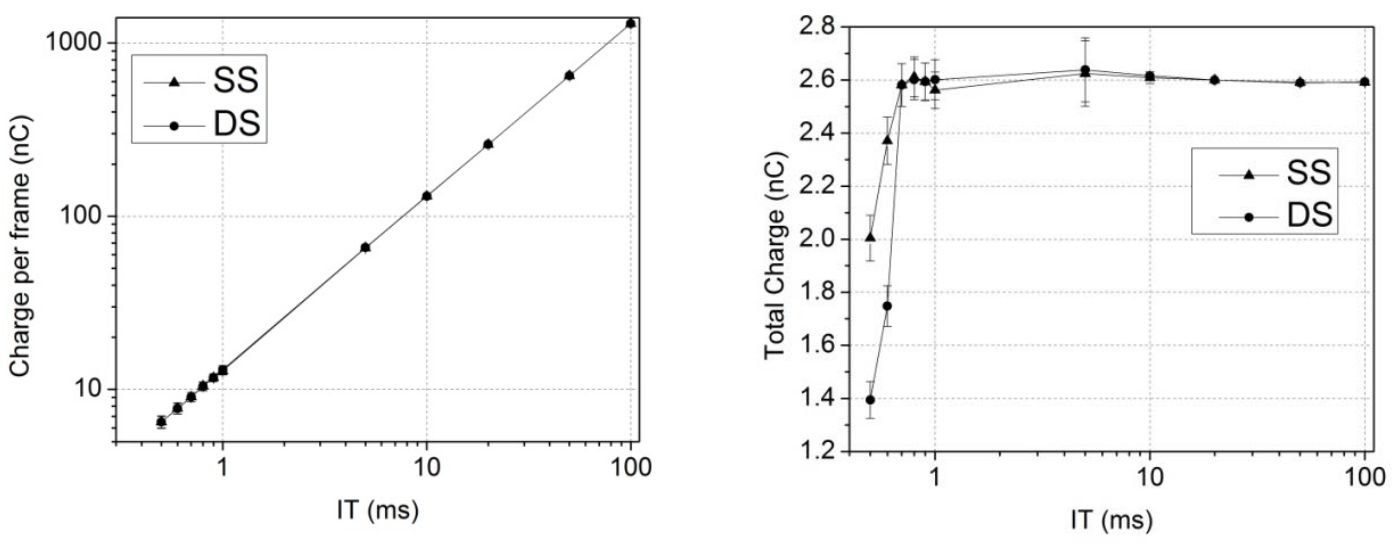

Figure 2. a-left) Charge measured in a single frame as a function of the IT (logarithmic scale); b-right) Total charge as a function of the IT in one second total acquisition time. Error bars have been calculated considering two standard deviations of the distribution of the response around the expected value.

\section{Experimental results and discussion}

TERA06 have been set to have a conversion factor of $200 \mathrm{fC}$ per count has suggested by the manufacturer for maximisation of the dynamic range. The possibility to use the TERA06 with two different sampling modalities (SS or DS) requires a test to compare the performance of the chip varying the integration time. For the purpose to measure the effect of the DS and SS especially at very short IT, a fixed current of $2.65 \pm 0.01 \mathrm{nA}$ has been generated by applying a fixed voltage across a set of 128 resistors, each $1 \mathrm{G} \Omega$. Figure 2a shows the charge measured in a single frame as function of the integration time. The chip has a good linearity of the response and there is an excellent agreement between the two modalities. Figure $2 \mathrm{~b}$ shows the total charge, obtained as the sum of all the frames, for different integration times. At high sampling frequency (IT below $1 \mathrm{~ms}$ ) there is a strong discrepancy between the measured and the expected total charge which is even more pronounced when the DS modality is used.

The sampling frequency of $1 \mathrm{kHz}$, corresponding to $1 \mathrm{~ms}$ of IT seems to be the higher limit for a reliable operation of the TERA06. The chip seems to be unresponsive for higher sampling frequencies with the digital output occasionally frozen. This effect is much more evident when the DS mode is adopted. In DS mode, the response for the n-frame is calculated by the subtraction of the counts of the (n-1) frame. If the digital output freezes there are occasionally frames which have null response and cumulating the response across the total acquisition time produces a lack of charge measured. Overall the two sampling modalities show comparable performances resulting on the selection of the SS mode as standard setup in order to decrease the computational load of the GUI.

Figure 3 shows the linearity of the DAQ B as a function of the integration time. The minimum selectable value is $14 \mu \mathrm{s}$, limited by the AFE0064, whilst the maximum value is limited only by the full scale range. The constant current adopted for the measurement corresponds to $18.3 \pm 0.02 \mathrm{nA}$. The response of the system is linear over all the dynamic range; the experimental measurement overestimates the expected value of $2 \%$ with an uncertainty produced by the noise fluctuation of $\pm 0.75 \%$. 


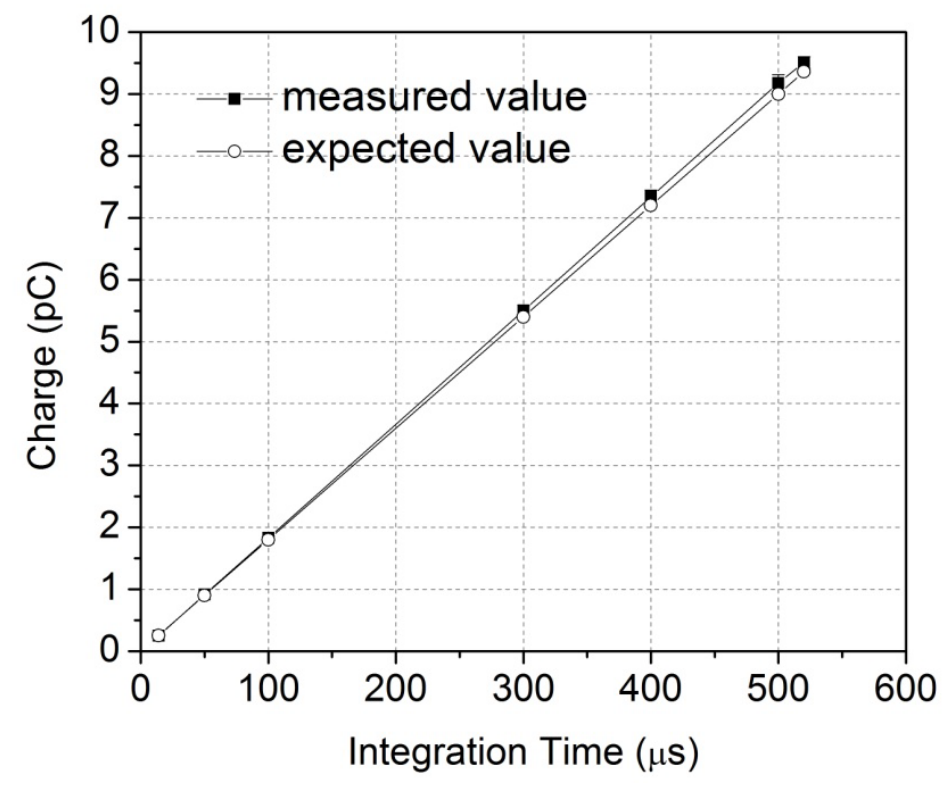

Figure 3: DAQ B linearity

The DAQ C (fig. 4) shows an excellent linearity as a function of the input current; the agreement between expected and measured values is within $0.88 \%$.
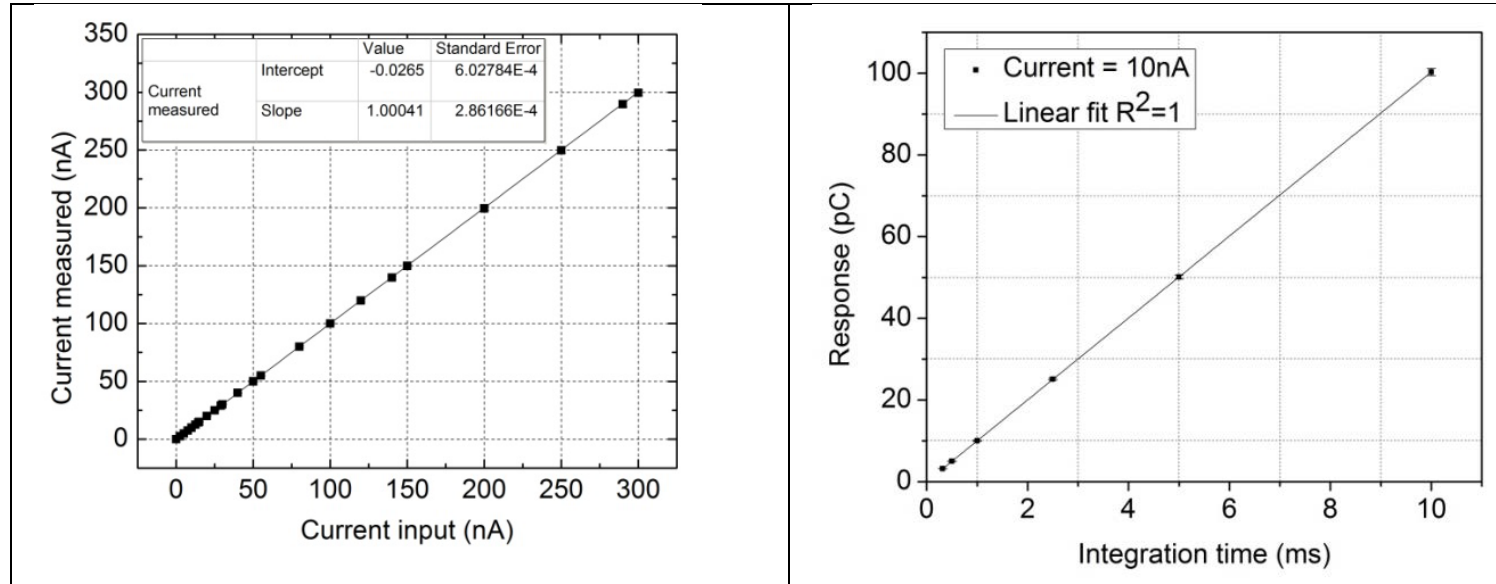

Figure 4: DAQ C linearity

Figure 5 summarises and compares the full scale ranges for the DAQ A, B and C as a function of the input charge. Data have been collected using the $1 \mathrm{G} \Omega$ resistors for DAQ A and $10 \mathrm{M} \Omega$ for DAQ B and C. By changing the voltage across the resistors and the integration time, each DAQ has been forced to measure from its lowest value up to the full scale. This characterisation is particularly useful to identify the specific range of use of these three frontends in radiotherapy quality assurance. By selecting the largest range for both the AFE0064 and the DDC264, corresponding to $9.6 \mathrm{pC}$ and $150 \mathrm{pC}$ full scale, respectively, the DAQ become complementary covering a dynamic range from less than $1 \mathrm{pC}$ up to tens $\mathrm{nC}$ including also the TERA06. Specially for applications such as stereotactic body radiotherapy where a massive amount of dose per fraction (20 Gy/f) [13] is delivered in a small area for treatment of tumours of brain, spinal cord and lung, a wide dynamic range DAQ with high temporal (fast acquisition 


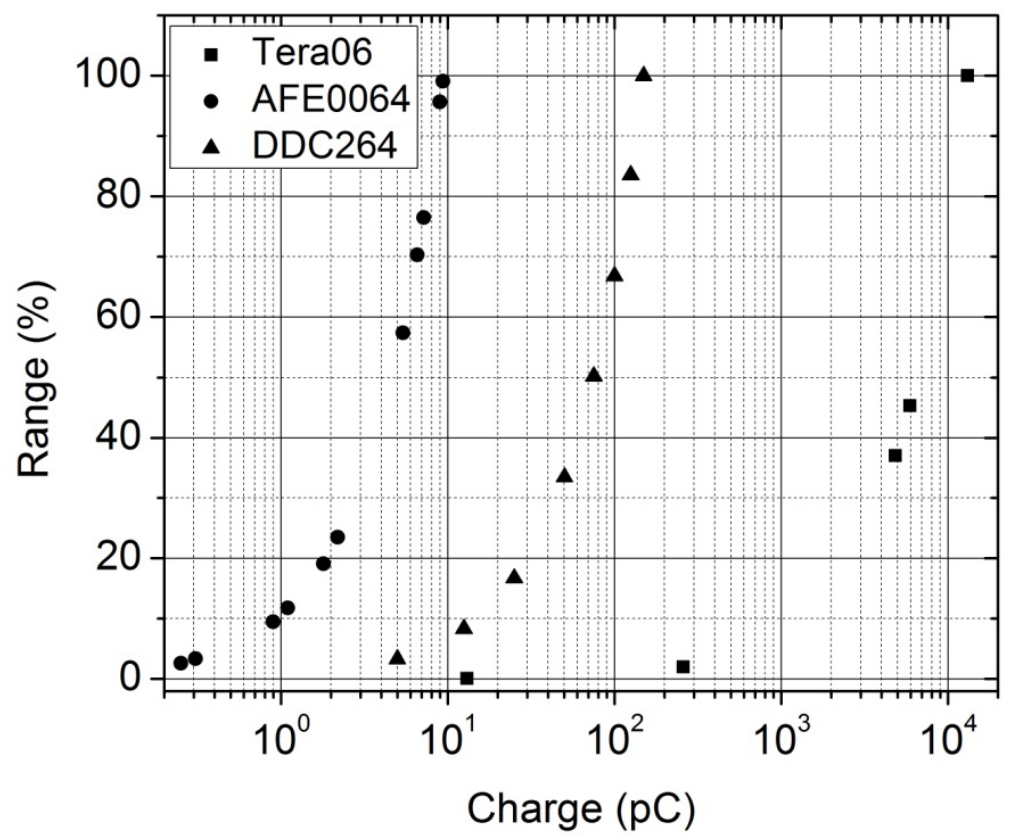

Figure 5: Linearity of the three Data Acquisition Systems

time) and spatial (large number of channels) resolution are stringent requirements for a quality assurance instrumentation. QA procedures also for standard Stereotactic Radiosurgery and IMRT treatments require dose profile at large depth where a high sensitivity and high spatial resolution are essential requisites of the detector DAQ.

Figure 6 shows the characterisation of the noise tolerance of the DAQ. The contribution of the thermal noise due to the resistors (1 G $\Omega$ for DAQ A and $10 \mathrm{M} \Omega$ for DAQ B and C) used for the calibration of the devices has been evaluated as function of the integration time and displayed against the noise acquired. The peak shown by the TERA06 front end at IT of $5 \mathrm{~ms}$ and $50 \mathrm{~ms}$ can be explained by the influence of the electromagnetic background noise (at 50 and $100 \mathrm{~Hz}$ ), when the period of the overlapping noise causes an increase of the standard deviation of the measured outputs. For longer IT instead, the background noise is averaged, as many periods are picked up and averaged. Even in the worst case, the contribution of the noise is less than $4 \%$ of the lowest charge measured by the TERA06 front end, so it does not compromises the performances of the DAQ. DAQ B and C show a linear increase of the noise versus the integration time. The detailed datasheets, provided by the manufacturer, predict the noticed behaviour with graphs showing a slightly linear increase for both the devices. The trend is emphasized by the resetting procedures, which do not take in account the duration of the IT, and by the contribution of the noise current integrated over a longer integration time which results in a higher baseline. At IT longer than $500 \mu$ s DAC B evidences the effect of the saturation: as the chip begins to saturate, the value read by the ADC is capped by the full scale value, thus decreasing the standard deviation of the value read. DAQ C instead does not seem to be sensitive to any trigger or electromagnetic noise interference due to its peculiarity to have an embedded digital interface which minimises the effects of noise perturbation of the weak analogue input signals. 

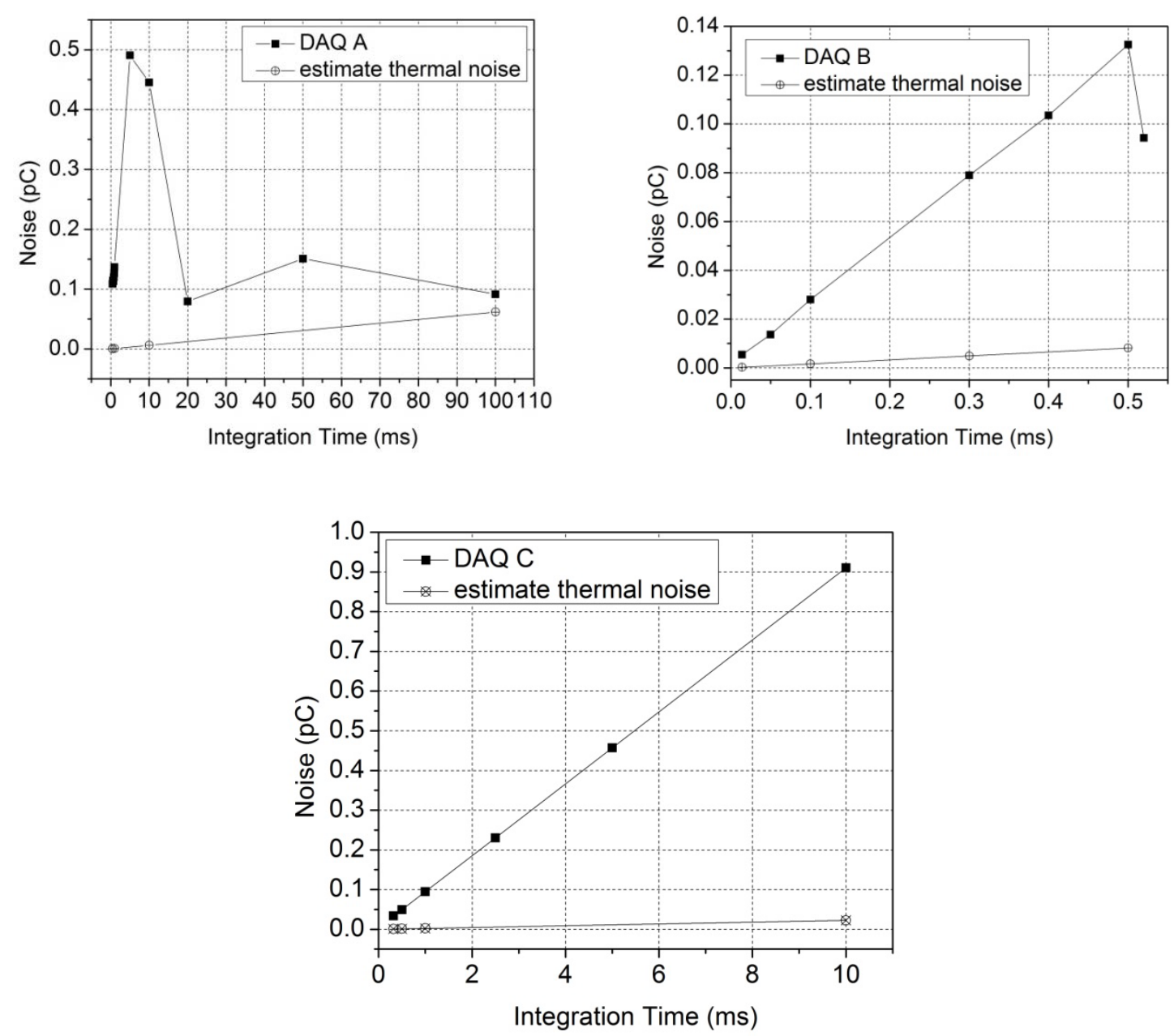

Figure 6: DAQ A, DAQ B and DAQ C noise characterization

\section{Conclusion}

All three front-ends present specific characteristics which make them suitable for different applications in QA for radiotherapy. Each DAQ is modular and can be easily adjusted to read multiple detectors with a large number of channels. DAQ A, based on the TERA06 chip is already used in dosimetry instrumentation to readout ionising chamber arrays up to 1024 channels and sensitive volume of the order of $0.13 \mathrm{~cm}^{3}$. Due to the procedure used by DAQ A for the data transfer, an increase of the number of channels causes an increase of the dead time of about $14 \mu$ s per additional chip, 64 channels each. A trade off between number of channels, acceptable dead time and computational and data handling load is necessary in order to achieve the best performances from the read-out front end. A new generation of detectors for QA in radiotherapy has been designed by the CMRP with high spatial resolution and small sensitive volumes based on pixellated silicon detectors (equivalent sensitive volume is approximately 0.1 $\mathrm{mm}^{3}$ ). The number of channels required for such devices is greater than 1024 and the gain of the front-end is very high to overcome a weak signal generated by the radiation into the small sensitive volume of the detector. To suit the new application, the DAQ B, based on the AFE0064 chip, was introduced and was proved to cover the range between $0.2 \mathrm{pC}$ and $10 \mathrm{pC}$ 
with integration time as small as $14 \mu$ s. The system is designed by a modular approach allowing combinations of 128, 256 or 512 channels modules with, virtually, no limitations in terms of number of modules readout in parallel. It shows a good linearity across the whole dynamic range and excellent timing performance with a sustainable sampling rate of $10 \mathrm{kHz}$. Data rate and death time of the system are not affected by increasing the number of channels because the DAQ B is designed adopting a parallel architecture to readout the modules. The gap of dynamic range and reliable integration time range left by the two architectures has been filled in by the DDC264, which has been successfully tested in terms of linearity and noise performance. It shows a dynamic range from approximately $5 \mathrm{pC}$ up to $150 \mathrm{pC}$ over an integration time ranging from $0.5 \mathrm{~ms}$ to $10 \mathrm{~ms}$. Despite the different internal architectures, the DAQ A and C show a comparable noise immunity with less than $0.5 \mathrm{pC}$ at $5 \mathrm{~ms}$ integration time, while the DAQ B is particularly good for very low noise applications with $0.13 \mathrm{pC}$ at $0.5 \mathrm{~ms}$ integration time which corresponds to $260 \mathrm{pA} / \mathrm{channel}$ at room temperature. The flexibility of the platform developed for this work, comprehends by the FPGA back-end interface (front-end data handler and USB2.0 interface), the software and host drivers, makes the design of a mixed analogue frontend architecture (for example DAQ B and C) feasible. The DAQ B and C have similar timing requirements (20 MHz clock frequency) and they can be easily interfaced to the FPGA by two parallel and hence independent, communication protocols based on chip specific control and data signals. This approach is convenient for applications where different channels must have different dynamic ranges. This is particularly true when the dose rate across the radiation field changes over few orders of magnitude and the detector array has sensitive elements with the same volume size (such as in Microbeam Radiation Therapy [14]) or for specific detector array where the sensitive volume of the sensor varies in dimensions to achieve a finer resolution in specific areas (such as for the semicircular array detector developed for dosimetry in brachytherapy of the melanoma eye cancer [15]).

\section{Acknowledgments}

This research project has been supported by the Australian National Health and Medical Research Council by the Project Grant \#1030159.

\section{References}

[1] N. Hardcastle, W. A. Tome', K. Foo, A. Miller, M. Carolan, and P. Metcalfe, Comparison of prostate IMRT and VMAT biologically optimized treatment plans, 2011 Med. Dosim. 3 Vol. 36. pp 292-298.

[2] http://www.ptw.de/2403.html. www.ptw.de. [Online] PTW Freiburg GmbH 2012, 8 29, 2012. [Cited: $125,2013$.

[3] http://www.sunnuclear.com/documents/arccheck.pdf. http://www.sunnuclear.com. [Online] [Cited: 04 24, 2013.]

[4] J.H.D. Wong et al., Characterization of a novel two dimensional diode array the "magic plate" as a radiation detector for radiation therapy treatment, 2012 Medical Physics 5 Vol. 39. pages 25442558.

[5] G .Mazza et al., A large dynamic range charge measurement ASIC family for beam monitoring in radiotherapy applications, 19-25 Oct 2008 Nuclear Science Symposium Conference Record pp 1077-1080.

[6] http://www.ti.com/lit/ug/sbau186/sbau186.pdf. www.ti.com. [Online] 03 2011. [Cited: 01 30, 2013.] SBAU186.

[7] P. Metcalfe, T. Kron, P. Hoban, The physics of radiotherapy x-rays and electrons, 2007 Medical Physics. 
[8] J. H. Wong et al, A silicon strip detector dose magnifying glass for IMRT dosimetry, 2010 vol. 37 No. 2 Medical Physics pp 427-439

[9] I. A. Pecka Valencia, Test and characterization of the beam monitor system for the Centro Nazionale di Adroterapia Oncologica. University of Torino, 2008. PhD Thesis.

[10] A. La Rosa et al., Characterization of a front-end electronics for the monitoring and control of hadrontherapy beam, 2008 Nuclear Instruments and Methods in Physics Research A pages 270-275

[11] http://www.ti.com/lit/ds/symlink/afe0064.pdf. www.ti.com. [Online] 09 2009. [Cited: 1 25, 2013.] SLA672.

[12] http://www.ti.com/lit/ds/symlink/ads8363.pdf. www.ti.com. [Online] 01 2011. [Cited: 01 30, 2013.] SBAS523B.

[13] S. H. Benedict et al,Stereotactic body radiation therapy: The report of AAPM Task Group 101, 2010 Medical Physics Vol. 37

[14] M. Petasecca et al., X-Tream: a novel dosimetry system for Synchrotron Microbeam Radiation Therapy, 2012, Journal of Instrumentation, 7, P07022, - doi:10.1088/1748-0221/7/07/P07022

[15] M. Weaver et al., Three-dimensional dosimetry imaging of I-125 plaque for eye cancer treatment, 2011, Nuclear Instruments and Methods in Physics Research, Section A, 633, SUPPL. 1, pp.S276S278 